\title{
QuANTIFYING THE MOTION OF MAGNETIC PARTICLES IN EXCISED TISSUE: EFFECT OF PARTICLE PROPERTIES AND APPLIED MAGNETIC FIELD
}

\author{
Sandip Kulkarni*1 ${ }^{1}$, Bharath Ramaswamy ${ }^{1}$, Emily Horton ${ }^{1}$, Sruthi Gangapuram ${ }^{1}$, Alek Nacev ${ }^{3}$, \\ Didier Depireux $^{2,4}$, Mika Shimoji ${ }^{1,4}$, Benjamin Shapiro ${ }^{1,2,4}$ \\ ${ }^{1}$ Fischell Department of Bioengineering, ${ }^{2}$ The Institute for Systems Research, \\ University of Maryland at College Park, MD 20742, United States. \\ ${ }^{3}$ Weinberg Medical Physics, LLC, ${ }^{4}$ Otomagnetics, LLC \\ *Corresponding Author: sandip.d.kulkarni@gmail.com
}

\begin{abstract}
This article presents a method to investigate how magnetic particle characteristics affect their motion inside tissues under the influence of an applied magnetic field. Particles are placed on top of freshly excised tissue samples, a calibrated magnetic field is applied by a magnet underneath each tissue sample, and we image and quantify particle penetration depth by quantitative metrics to assess how particle sizes, their surface coatings, and tissue resistance affect particle motion. Using this method, we tested available fluorescent particles from Chemicell of four sizes $(100 \mathrm{~nm}, 300 \mathrm{~nm}, 500 \mathrm{~nm}$, and $1 \mu \mathrm{m}$ diameter) with four different coatings (starch, chitosan, lipid, PEG/P) and quantified their motion through freshly excised rat liver, kidney, and brain tissues. In broad terms, we found that the applied magnetic field moved chitosan particles most effectively through all three tissue types (as compared to starch, lipid, and PEG/P coated particles). However, the relationship between particle properties and their resulting motion was found to be complex. Hence, it will likely require substantial further study to elucidate the nuances of transport mechanisms and to select and engineer optimal particle properties to enable the most effective transport through various tissue types under applied magnetic fields.
\end{abstract}

\section{Introduction}

In the fields of magnetic drug targeting ${ }^{1}$, hyperthermia ${ }^{2}$, and magnetic resonance imaging ${ }^{3,4}$ there is a need to be able to transport magnetic nanoparticles (MNPs) to desired tissue locations. How MNPs move in-vivo depends on their properties, the properties of the surrounding biological milieu, and on the strength of the applied magnetic field gradient. In prior work, we analyzed how the transport of MNPs in blood vessels depends on particle constitution, size, the velocity and profile of blood flow in vessels, and the strength of the applied magnetic field gradient ${ }^{5,6}$. In this article, we begin to address the second half of the question: how MNPs move through the tissue between blood vessels. By measuring how particle motion in freshly excised tissue depends on particle size, coating, tissue type, and the applied magnetic field gradient, we hope to provide researchers with tools for better understanding that motion in order to help select MNP designs to improve therapy ${ }^{1,7-13}$ and diagnosis ${ }^{4,13-17}$.

Substantial evidence indicates that particle characteristics (size, surface chemistry, volume of magnetic content) influence their motion through biological media such as mucus ${ }^{18}$, liquids and gels ${ }^{19}$, and brain 
tissue $^{10,11}$. In mucus ${ }^{18}$, modifying particle size and coating led to 10 fold and 10,000 fold changes in diffusion respectively. In liquids and gels ${ }^{19}$, among a set of particles $(10 \mathrm{~nm}$ and $50 \mathrm{~nm}$ size, uncoated, polystyrene coated and dextran coated), only $50 \mathrm{~nm}$ dextran coated particles moved, and movement in glycerol was 20 times faster than in collagen gel. In brain tissue, the width of extracellular spaces (30-64 $\mathrm{nm})$ precludes liposomes above $100 \mathrm{~nm}$ from penetrating the brain during convection enhanced delivery $^{20-22}$. Particle steric coating (e.g. polyethylene glycol or PEG) and charge also influence binding to cells and thereby limit or improve diffusion in brain tissue ${ }^{20,21,23}$.

To our best knowledge, as yet there have been no experimental studies that have quantified the ability of magnetic forces to transport different types of MNPs through different tissue types. Prior research has included the study of MNP motion in liquids ${ }^{19,24}$ and gels ${ }^{19}$ and indicated that MNP penetration depends strongly on the characteristics of the particles and the surrounding medium. Motion of magnetic beads within in vitro cultured cells (fibroblasts and actins) has been studied for measurement of cytoplasmic viscosity and motility ${ }^{25}$; however, the focus was on rheology within cells rather than passage of MNPs through tissue. Motion of particles in cells was also studied in Zhang et al. ${ }^{26}$ to create rotation and apoptosis of cells. MNP penetration in ex-vivo human skin was studied qualitatively in Baroli et al. ${ }^{27}$ but only for passive diffusion.

Several forces are thought to influence the motion of MNPs in tissue ${ }^{28}$. The magnetic force $\left(\mathrm{F}_{\mathrm{MAG}}\right)$ is the force applied by the external magnet on the particles. Inter-particle interaction forces ${ }^{29}$ act on magnetized particles and can lead to their agglomeration ${ }^{29-31}$. Tissue resistance $\left(\mathrm{F}_{\mathrm{TR}}\right)$ is likely composed of a viscous drag force $\left(\mathrm{F}_{\mathrm{D}}\right)$ and the binding force $\left(\mathrm{F}_{\mathrm{S}}\right)$ due to the adhesion between particle surface coatings and the tissue microenvironment ${ }^{32-37}$. The contribution of these component forces and the interplay between them is complex and has been a challenge to address. As far as we know, there are no accepted mathematical theories available for adequately capturing these complexities. Hence, in this article we focus on experimentally measuring particle movement in tissue samples.

We present a simple experimental technique to quantify the motion of magnetic particles through tissue. To arrive at these first results to understand and quantify particle motion in tissue under the influence of magnetic fields, we used freshly excised tissue (ex-vivo experiments). Even though we took sensible precautions to ensure that our excised tissue samples remained as close in their properties to living tissue as possible (excision time was less than two hours, tissue was preserved in a cool environment and was stored in phosphate buffer solution), we note that our collected results may still differ from in-vivo particle behavior because even with optimal procedures excised tissue is known to differ from live tissue $^{38-40}$. In our approach, fluorescent MNPs were placed on top of freshly excised tissue samples and a magnetic field was applied by placing a magnet under the tissue. After a set time, the tissue was fixed (flash-frozen), sliced, imaged using a fluorescent microscope, and particle movement was then quantified by processing the three-dimensional volume of particles from stacked images using standardized quantitative metrics. Experiments conducted with and without a magnetic field distinguished the effect of diffusion versus magnetic drift. Our results indicate how particle properties and tissue types can affect particle motion, under what circumstances the magnetic field is most effective at moving particles in tissue, and which particle types among those tested should be selected for efficient magnetic transport.

\section{Experimental Methods}

We developed and implemented a methodology to measure the rate of MNP movement in fresh tissue. To do so, we placed fluorescent MNPs on top of freshly excised rat tissue and applied a high magnetic field gradient by placing a permanent magnet at a precise distance underneath the MNPs below the tissue. This magnet moved the MNPs into and partway through the tissue. The tissue was then fixed and a 3dimensional cryostat imaging system similar to Shen et $\mathrm{al}^{41}$ sliced the tissues and imaged the distribution 
of particles in the tissue samples. The acquired imaged data was pruned and the images were stacked into a 3-dimensional volume. Standardized metrics representing the degree of particle penetration into tissue samples were used to quantitatively assess the depth of particle penetration into the tissue. Then penetration depth was tabulated to compare the effects of particle size (hydrodynamic diameter), surface coating, tissue type (brain, liver, kidney), and magnetic field gradient on MNP motion in tissue. A flow diagram of this process is shown in Fig. 1 below.

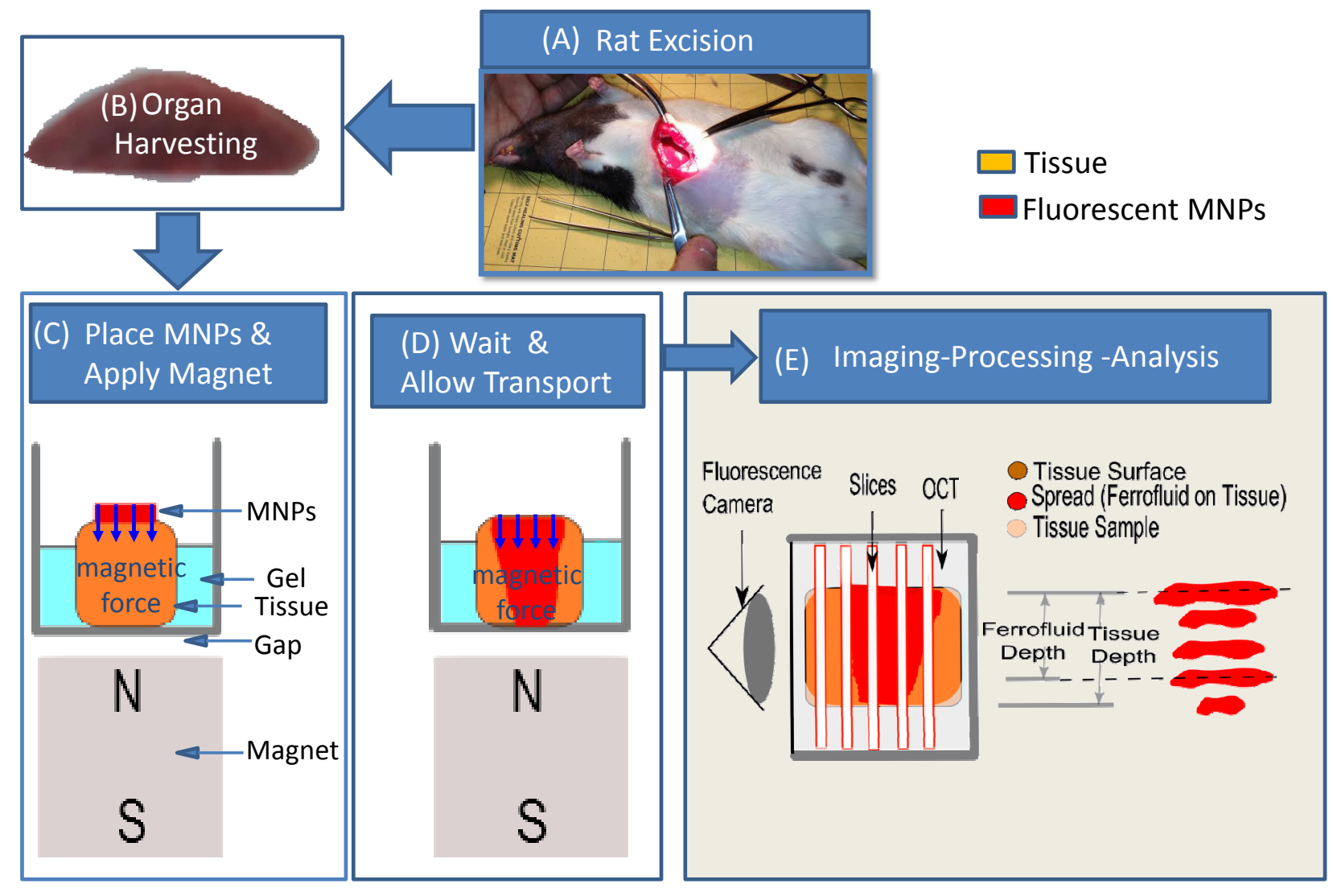

Fig. 1. A schematic of the experimental procedure. A) Excision of an organ from a rat. B) Excised tissue. C) Magnetic nanoparticles (MNPs) were placed on top of the tissue sample in solution (as a ferrofluid). The permanent magnet was then applied at a prescribed distance below the tissue sample to create a calibrated magnetic force on the particles (see placement calibration illustrated in Figure 3). D) Resulting distribution of particles in tissue sample after 45 minutes. E) The tissue was fixed in OCT (optimal cutting temperature fluid) and then sliced and imaged using an automated cryostat and a fluorescence camera. The penetration depth of the ferrofluid was then measured and quantified by a standardized metric.

\subsection{Materials and Preparation}

Long Evans Rats (obtained from Charles River) were used to obtain the tissue samples. The rats were anesthetized using Isoflorane gas and then sacrificed. All surgical and experimental procedures were approved by the University of Maryland Animal Care and Use Committee and were in accordance with NIH Guidelines on the care and use of laboratory animals.

Freshly excised organs were stored in phosphate buffer (PBS) at $4{ }^{\circ} \mathrm{C}$ for about $1-2$ hours until the experiments. Tissue sections of 4-6 mm thickness were prepared, embedded in liquefied gelatin in a 10 $\mathrm{mm}$ petri dish, and then cooled at 4 degrees until the tissue was immobilized. 
A variety of fluorescent magnetic nanoparticles (MNPs) purchased from Chemicell GmbH were used for our experiments. In this first study, we elected to use particles from Chemicell because we have used Chemicell particles for many years, and are familiar with the handling, properties and behaviors of these particles. Four available sizes $(100 \mathrm{~nm}, 300 \mathrm{~nm}, 500 \mathrm{~nm}$ and $1 \mu \mathrm{m})$ and four coatings (Chitosan, Starch, Lipid and PEG/P) were selected. Chemicell $\mathrm{GmbH}$ provided us with two types of particles. A) NanoscreenMag particles with hydrodynamic size $<300 \mathrm{~nm}$ and with the fluorescent dye outside the iron core beneath the external coating; B) ScreenMag (or SiMag) particles with hydrodynamic size between $500 \mathrm{~nm}-1 \mu \mathrm{m}$ where the fluorescent dye is incorporated in a silica shell around the core and the particle coating is around the silica shell. All particles contained a fluorescent red dye (lipophilic fluorescence dye Lumigen-Red for nanoscreenMAG and nil-blue for screenMAG) for easy visualization of the distribution of the particles in tissue. These two types of particles are illustrated in Figure 2. In our experiments, the dye was excited at $578 \mathrm{~nm}$ and emission was measured at $613 \mathrm{~nm}$ to quantify the distribution of particles in the tissue samples. Chemicell was unable to synthesize starch particles $>500 \mathrm{~nm}$, lipid particles $>300$ $\mathrm{nm}$ and PEG/P particles $>200 \mathrm{~nm}$ due to their higher molecular weight (these particles did not remain stable at the larger sizes). Our tissue experiments were conducted for those fluorescent particles that were available from Chemicell.

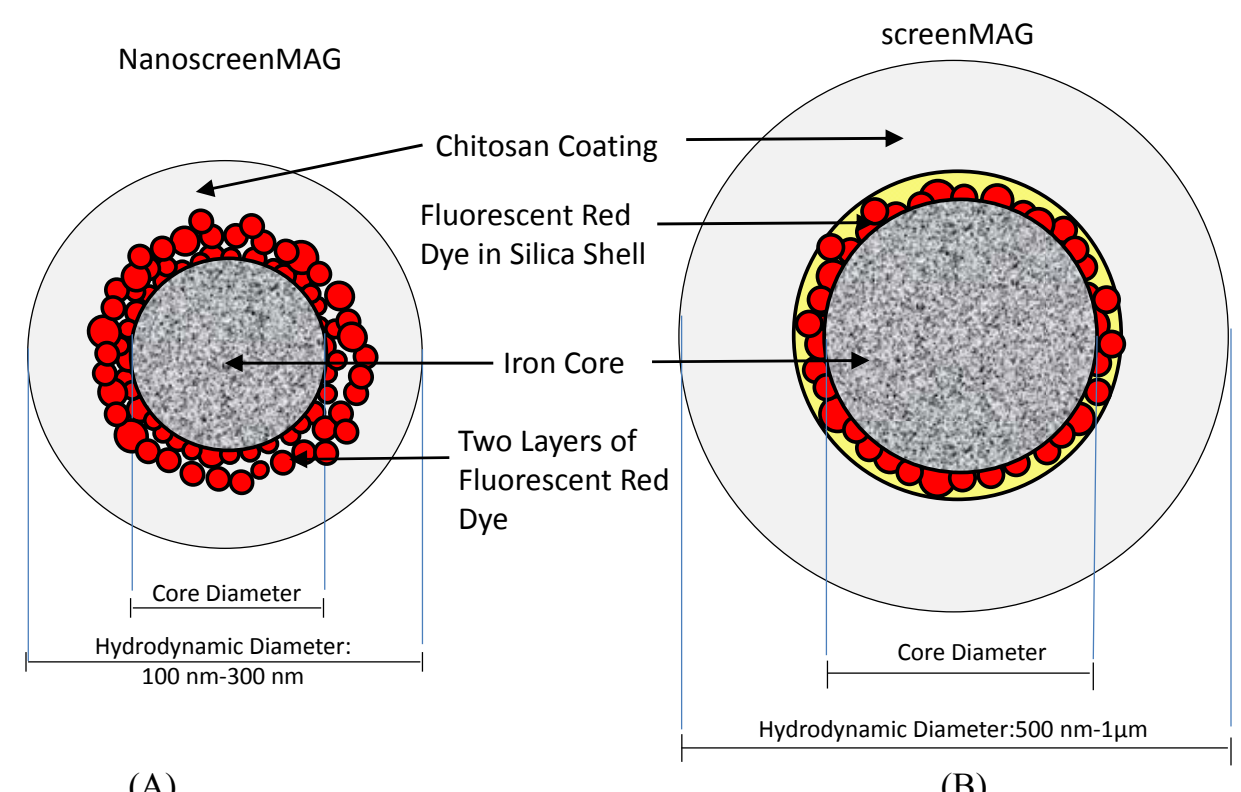

(A)

(B)

Fig. 2. Illustration of the structure of the Chemicell particles employed in this study. A) NanoscreenMag particles contain two red fluorescent layers around the iron core but inside the particle coating. B) In the ScreenMag particles, the fluorescent dye is incorporated into a silica shell and the particle coating is outside this shell. In both cases, the fluorescent dye enabled visualization of the particle distribution inside tissue samples but is not in direct contact with the tissue.

To keep the experimental setup simple and compact, we employed small $\mathrm{NdFeB}$, grade $\mathrm{N} 42$, permanent bar magnets with Nickel-Copper-Nickel triple layer coated, size 1" x 1" x 2", magnetized through the 2 inch length with poles on $1 \mathrm{x} 1$ inch surfaces (from Applied Magnets Inc.). These magnets had a high field strength (surface field strength of $\sim 0.4 \mathrm{~T}$ and core strength of $1.5 \mathrm{~T}$ ), which created a strong maximum magnetic field gradient $(\sim 30 \mathrm{~T} / \mathrm{m})$. The magnets were placed below the tissue blocks as shown in Figure $1 \mathrm{C}$ in order to effectively pull particles towards the magnet. We found that these magnets applied a sufficient magnetic field gradient to effectively move the different types of MNPs through the tissue samples. The magnets were small enough to permit a convenient experimental setup but big enough to enable careful calibration of the magnetic forces applied to the particles (discussed next). 


\subsection{Magnetic Field and Force}

The magnetic force we applied on the MNPs was calibrated by measuring the magnetic field and gradient around each permanent magnet. A single ferromagnetic particle will experience a magnetic force $\vec{F}=k M[D \vec{H} / D \vec{x}]=k \vec{M} \nabla \vec{H}^{5,7,42-44}$ where $\vec{H}$ is the applied magnetic field, $\vec{M}$ is the resulting magnetization of the particle, $[D \vec{H} / D \vec{x}]$ is the Jacobian spatial derivatives matrix, $\nabla$ is the spatial gradient, and $k$ is a constant that depends on particle properties. Here the first and second expressions are equivalent by the chain rule and it can be seen that the force on a MNP depends on both the particle magnetization $\vec{M}$ and the applied magnetic field gradient $\nabla \vec{H}$. In our experiments, the applied magnetic field was strong enough to saturate the particles hence $\|\vec{M}\|$ achieved its maximal strength of approximately $3.2 \mathrm{emu} / \mathrm{g}$. Each magnet was placed underneath the tissue samples at a location, which produced a roughly constant magnetic motive force on the particles as they moved through the tissue, samples (please see Figure 3).

First, the magnetic field surrounding the bar magnets was measured using a Lakeshore 460-3 Channel Gaussmeter with a measurement range from $0.03 \mathrm{mT}$ to $30 \mathrm{~T}$. The device has a Hall probe (MMZ-2518$\mathrm{UH}$ ) encased in a protective brass sleeve attached to three orthogonal unislide components (from Velmex) forming a 3-D stage. The stepper motors controlling the stages have an internal step monitor for relaying signals via serial connection to a computer. The stepper motors have a resolution of 400 steps per revolution, with a single step corresponding to a displacement of $6.34 \mu \mathrm{m}$ along any of the three axes (orthogonal directions). The 3-D stage can be controlled and sensed using a GPIB IEEE488 and RS232 connections to Labview interface. The magnetic field around each permanent magnet was measured using this system.

Second, the above measurements allowed accurate calculation of the $\nabla \vec{H}$ value at each location around the magnet, and hence a good estimate of the strength of $\vec{M} \nabla \vec{H}$ that would be applied to particles at that location. The magnetic saturation value $\|\vec{M}\|$ was measured by vibrating sample magnetometry (VSM) as detailed in Sec. 2.3.1. The resulting calculated spatial variation of $\|\vec{M} \nabla \vec{H}\|$ around the bar magnets is shown in Fig. 3 below. Based on this data, in all experiments the top face of the bar magnet was placed $6 \mathrm{~mm}$ below the MNPs to ensure that all particles experienced a starting motive force proportional to $\|\vec{M} \nabla \vec{H}\|=3.9 \pm 2 \times 10^{8} \mathrm{~A}^{2} / \mathrm{m}^{3}$. As the MNPs moved through the $\sim 6 \mathrm{~mm}$ width of the tissue samples, $\|\vec{M} \nabla \vec{H}\|$ could potentially increase up to $\|\vec{M} \nabla \vec{H}\|=8 \pm 4.1 \times 10^{8} \mathrm{~A}^{2} / \mathrm{m}^{3}$, thus the applied magnetic motive force varied by at most a factor of two as the MNPs moved through the tissue samples. In Table 1, the coefficient $\mathrm{k}$ varies with particle sizes. Magnetic force can be calculated as product of $\mathrm{k}$ and $\|\vec{M} \nabla \vec{H}\|$. 


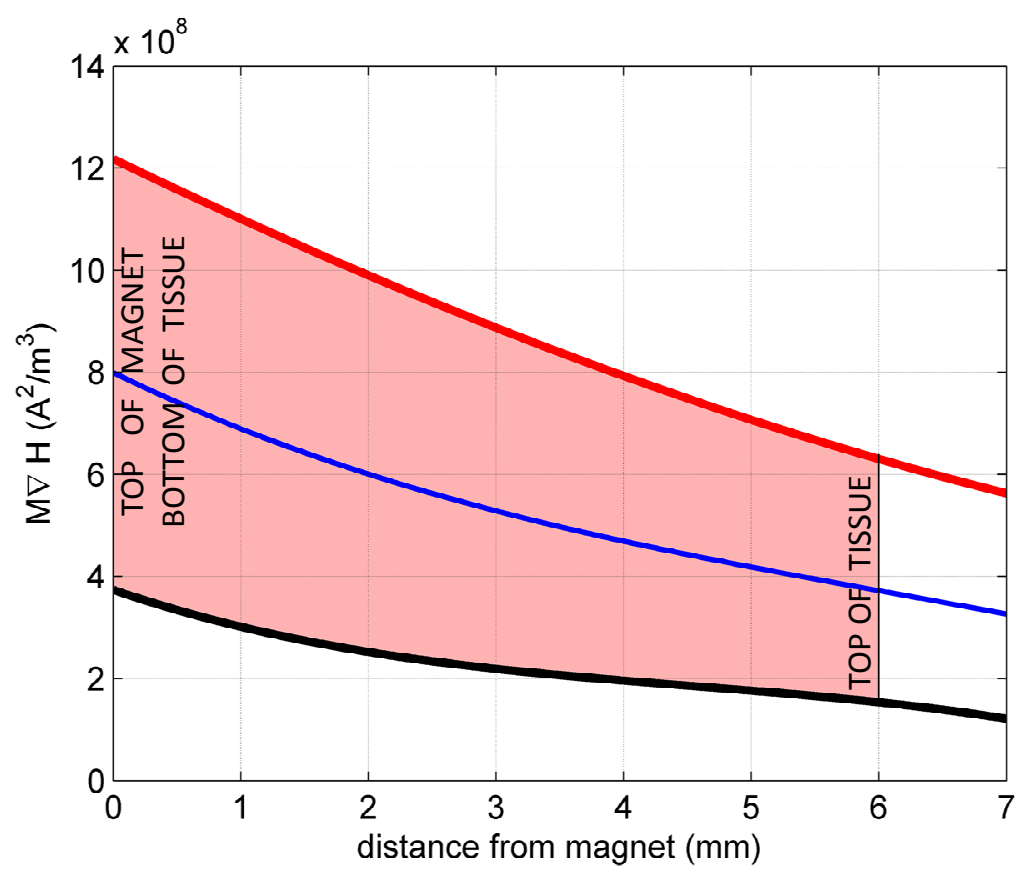

Fig. 3. Measured and calculated variation of the magnetic motive force on the magnetic particles as they moved through the tissue samples. The strength of the magnetic motive force is proportional to $\|M \nabla \vec{H}\|$, which is plotted above versus particle distance from the magnet (in units $\mathrm{A}^{2} / \mathrm{m}^{3}$ ). The red line indicates the estimated maximum, the black is the estimated minimum, and the blue line is the average value. Based on this data, we chose the magnet size in order to, as much as possible, apply a fairly uniform motive force across the entire tissue sample. As shown, the motive force increases by at most a factor of two from the top of the tissue $(6 \mathrm{~mm}$ away from the top of the magnet) to the bottom of the tissue (right above the magnet surface).

Table 1 Approximate $k$ values, for particles of various size, listed in units of $N /\left(A^{2} / m^{3}\right)$. The coefficient $k$ relates the quantity $\|M \nabla \vec{H}\|$ shown above in Figure 3 with the actual magnetic force on the particle by $\|\mathbf{F} \mid=\mathbf{k}\| M \overrightarrow{\boldsymbol{H}} \|$.

\begin{tabular}{ccccc}
\hline & \multicolumn{4}{c}{ Particle Type (Size) } \\
\cline { 2 - 4 } & $100 \mathrm{~nm}$ & $300 \mathrm{~nm}$ & $500 \mathrm{~nm}$ & $1000 \mathrm{~nm}$ \\
\hline $\mathrm{k}$ & $\approx 0.0007 \times 10^{-24}$ & $\approx 0.0178 \times 10^{-24}$ & $\approx 0.082 \times 10^{-24}$ & $\approx 0.658 \times 10^{-24}$ \\
& $\mathrm{~N} /\left(\mathrm{A}^{2} / \mathrm{m}^{3}\right)$ & $\mathrm{N} /\left(\mathrm{A}^{2} / \mathrm{m}^{3}\right)$ & $\mathrm{N} /\left(\mathrm{A}^{2} / \mathrm{m}^{3}\right)$ & $\mathrm{N} /\left(\mathrm{A}^{2} / \mathrm{m}^{3}\right)$ \\
\hline
\end{tabular}

\subsection{Properties of the Tested Magnetic Nanoparticles (MNPs)}

Motion of MNPs through tissue depends upon their properties, such as their saturation magnetization, size, and surface charge. Before measuring the motion of particles through tissue samples, we first characterized the properties of each particle type using available measurement techniques. We used vibrating sample magnetometry (VSM) to measure saturation magnetization of particles, dynamic light scattering (DLS) to measure the size of the particles, and we further classified particles based on zeta potential measurements from Chemicell $\mathrm{GmbH}$. 


\subsubsection{Saturation Magnetization of the MNPs}

The magnetic force on particles is proportional to the saturation magnetization of the particles and the gradient of the applied magnetic field intensity $5,7,28,42-47$. We measured the saturation magnetization for each particle type using a vibrating sample magnetometer (VSM $)^{48}$. Table 2 shows magnetization values for each particle type, for the entire volume used $(4 \mu \mathrm{l})$ and also estimated per particle (as in the specification sheets from Chemicell, we divided the sample volume by the number of particles per volume to arrive at a per particle value). As expected, the per particle magnetization increases with particle size.

Table 2: Measured saturation magnetization values for the particles tested. For each particle size, the top entries are the measured value for the entire $4 \mu \mathrm{l}$ volume sample (in milli-emu electromotive units), middle entries are measured values in $\mathrm{emu} / \mathrm{g}$, and the bottom entries are the estimated per particle values. N/A (not available) is for particles types that could not be synthesized by Chemicell.

\begin{tabular}{|c|c|c|c|c|c|c|}
\hline \multicolumn{2}{|c|}{ Size $(\mathrm{nm})$} & Chitosan & PEG & Lipid & Starch & Mean \pm Standard \\
\hline \multirow{3}{*}{100} & memu per $4 \mu \mathrm{l}$ & 11.2 & 13.3 & 11.7 & 14.3 & $12.6 \pm 1.4$ \\
\hline & $\mathrm{Emu} / \mathrm{g}$ & 112 & 133 & 117 & 143 & $126 \pm 14$ \\
\hline & memu per particle & $6.2 \times 10^{-11}$ & $7.41 \times 10^{-11}$ & $6.5 \times 10^{-11}$ & $8 \times 10^{-11}$ & $7 \times 10^{-11} \pm 8 \times 10^{-12}$ \\
\hline \multirow{3}{*}{300} & memu per $4 \mu l$ & 10 & N/A & 8 & 4.33 & $7.4 \pm 2.8$ \\
\hline & $\mathrm{Emu} / \mathrm{g}$ & 100 & N/A & 80 & 43.4 & $74 \pm 28$ \\
\hline & memu per particle & $1.5 \times 10^{-09}$ & $\mathrm{~N} / \mathrm{A}$ & $1.22 \times 10^{-09}$ & $6.6 \times 10^{-10}$ & $1 \times 10^{-09} \pm 4 \times 10^{-10}$ \\
\hline \multirow{3}{*}{500} & memu per $4 \mu \mathrm{l}$ & 7.9 & $\mathrm{~N} / \mathrm{A}$ & $\mathrm{N} / \mathrm{A}$ & 7.3 & $7.6 \pm 0.4$ \\
\hline & $\mathrm{Emu} / \mathrm{g}$ & 79 & N/A & N/A & 73 & $76 \pm 4$ \\
\hline & memu per particle & $5.2 \times 10^{-09}$ & $\mathrm{~N} / \mathrm{A}$ & $\mathrm{N} / \mathrm{A}$ & $4.9 \times 10^{-09}$ & $5.1 \times 10^{-09} \pm 2.5 \times 10^{-10}$ \\
\hline \multirow{3}{*}{1000} & memu per $4 \mu l$ & 6 & N/A & N/A & $\mathrm{N} / \mathrm{A}$ & N/A \\
\hline & $\mathrm{Emu} / \mathrm{g}$ & 60 & N/A & N/A & N/A & N/A \\
\hline & memu per particle & $3.3 \times 10^{-08}$ & N/A & N/A & $\mathrm{N} / \mathrm{A}$ & $\mathrm{N} / \mathrm{A}$ \\
\hline
\end{tabular}

\subsubsection{Size Measurements of MNPs}

The hydrodynamic diameter of the MNPs was measured by dynamic light scattering, as employed previously in Murdock et al ${ }^{49-51}$. We employed a dynamic light scattering system from Photocor Instruments and found that the measured hydrodynamic measurements closely matched the specification provided by Chemicell GMBH. In most cases, we found that the peak of size distributions were within \pm $50 \mathrm{~nm}$ of the radius of values listed in the particle specification sheets. However, it was found that some particles were highly polydispersed, i.e. more than one peak for the particle size distribution (e.g. for 100 $\mathrm{nm}$ PEG/P particles). The poly-dispersity and agglomeration of $100 \mathrm{~nm}$ PEG/P and $300 \mathrm{~nm}$ lipid particles led to a higher measurement of hydrodynamic radius. All other particles were tightly distributed around the specified values as can be seen from Table 3 below. Detailed size distribution plots are further provided in the Supplementary Materials section. 
Table 3. Measurements of the hydrodynamic radius of Chemicell MNPs using the DLS (dynamic light scattering) system from Photocor Instruments shows that most particles comply with the particle sizes specified by Chemicell. However, the PEG/P and lipid particles were found to have poly-dispersity and agglomeration.

\begin{tabular}{|c|c|c|c|c|c|c|c|c|}
\hline & \multicolumn{2}{|c|}{$100 \mathrm{~nm}$} & \multicolumn{2}{|c|}{$300 \mathrm{~nm}$} & \multicolumn{2}{|c|}{$500 \mathrm{~nm}$} & \multicolumn{2}{c|}{$1 \mu \mathrm{m}$} \\
\cline { 2 - 9 } & Mean & Polydispersity & Mean & Polydispersity & Mean & Polydispersity & Mean & Polydispersity \\
\hline Chitosan & 49.9 & 0.99 & 119 & 0.528 & 267 & 0 & 524 & 1.08 \\
\hline Starch & 45 & 1.34 & 116 & 0.77 & 254 & 0 & & \\
\hline Lipid & 78 & 0.06 & 257 & 0.67 & & & & \\
\hline PEG/P & 131 & 0.8 & \multicolumn{7}{|c|}{} & \multicolumn{3}{|c|}{} \\
\hline
\end{tabular}

\subsubsection{Zeta Potential of MNP Coatings}

The ability of the MNPs to repel each other and remain dispersed in solvent is determined by their zeta potential $^{52-54}$. Zeta potential is the charge on the outer liquid layer of the particle. Stronger charges (positive or negative) keep the particles more dispersed. Hence the zeta potential determines the initial state of the particles and can alter the rate of aggregation of particles and hence potentially their ability to move through tissue. For the experiments in this paper, we consider four types of particle coatings: chitosan, polyethylene glycol (with a phosphate group that led to negative charge), lipid and starch. The stated zeta potential of each type of coating is provided in Table 4. These values were obtained from Chemicell GmbH.

Table 4: Zeta potential values for each particle type and coating in $\mathrm{mV}$, measured in double distilled $\mathrm{H}_{2} \mathrm{O}$ (medium of ferrofluid).

\begin{tabular}{lllll}
\hline \hline & Chitosan & PEG & Lipid & Starch \\
\hline \hline Charge Potential & +34 & -20 & -19 & -8 \\
\hline
\end{tabular}

\subsection{Procedure}

For each experiment, a known volume of fluorescent MNPs (a 4 microliter droplet that stayed within the profile area of the tissue) was placed on top of a tissue surface. The tissue had been immobilized using gelatin in a petri-dish. The petri dish was then immediately placed on top of a permanent magnet, for 45 minutes. The tissue was placed directly above the magnet so that the maximum magnetic field was acting on the particles. As described earlier, the magnet size had been selected so that this motive force would not change too greatly as the particles traversed the tissue samples. After magnet application, the tissue was immediately fixed by flash-freezing in liquid nitrogen. The frozen tissue was then stored at $-80^{\circ} \mathrm{C}$. The fixed tissue samples were then embedded in optimal cutting temperature (OCT) fluid from TissueTek Inc., for simultaneous slicing and imaging using an automated cryostat imaging system previously described in Shen et al. $^{41}$. The automated cryostat has a single fluorescent camera with a resolution of 35 $\mu \mathrm{m}$ per pixel that captures bright field and fluorescent images of each tissue slice and the fluorescence distribution of particles within that slice. The complete stack of images of all tissue slices provided the entire three-dimensional distribution of fluorescent MNPs in that tissue sample. The slicing plane for the imaging was chosen to be parallel to the planes in which the particles moved from the top of the tissue towards the magnet at the bottom (see Figure 1). The collected images provided quantitative information on the depth of penetration of the MNPs, as discuss next. 


\subsection{Post-Processing of Images}

In order to assess the depth of MNP penetration into tissues, we analyzed the images acquired by the automated cryostat. For each slice of tissue, the fluorescent camera captures two images: one bright field image showing tissue and background and one fluorescent image showing only particles. Each image captured by the camera is stored as a matrix of pixels in unsigned integer 16 format. In case of bright field images, a zero value of the pixel represents a black color and the maximum value of $2^{16}$ represents a white color. Similarly, in the case of fluorescent field images, a maximum pixel value represents bright fluorescence while 0 represents the absence of fluorescence and particles. The images acquired by the fluorescent camera of the automated cryostat required substantial processing in order to reliably extract a distance metric. The processing of images involved the following steps:

A. Data Cleaning: The automated cryostat slicing generated some random images along with useful images, due to electrical noise and sometimes due to overlapping of a previous slice with the current slice. To remove all spurious images, we stacked all image data for all slices and formed a vector whose every element was the total intensity of each slice/image. Since good images have tissue and OCT region, and since OCT is white (white represents high grayscale intensity values close to $2^{16}$ ) while tissue is dark gray (black represents low grayscale intensity values close to zero), we could easily filter out bad images using a median filter. Example good and bad images are shown in Fig. 4A.

B. Image Alignment: The tissue-OCT sample was sometimes not exactly aligned in the desired topto-bottom orientation due to error in placing the sample on the cryostat slicer. All tissue images were rotated until the top surface (indicated as a red line in Fig. 4B) was horizontal to within \pm 1 degree.

C. Cropping for Image Processing: We then cropped the first image of each sample until only the dark gray tissue region and some surrounding white OCT regions were visible in the image. This cropped region was used for all slices of the corresponding sample. This enabled further image processing.

D. Image Thresholding: Based on bright field images and their pixel intensities, an image intensity histogram was plotted and a threshold of intensity was selected such that the dark and bright regions could be clearly demarcated. Then, every region other than the tissue region was masked by assigning pixel values of zero.

E. Assigning Regions of Interest: Since the fluorescent image and bright field image are spatially co-registered, we masked the same region in the fluorescent image that was outside the tissue region (as in step D above) by assigning pixel values of zero. As a result, only regions inside tissue have non-zero pixel intensities. This was done for all slices to ensure that only the tissue region was considered for each particle depth measurement.

F. Total Fluorescence Intensity Vector along Tissue Depth: Each vertical tissue image (slice) is represented by a matrix of pixel intensities with each pixel corresponding to a $35 \mu \mathrm{m} \times 35 \mu \mathrm{m}$ area. The degree of fluorescence is correlated to the amount of particles in that pixel. To reduce computational burden, the intensities of particle concentrations along each horizontal row of this matrix were summed up. These sums were collected into a single vertical vector $\vec{i}=\left[i_{1}, i_{2}, \ldots, i_{n}\right]$ each of whose elements is the net particle intensity at that tissue depth for this single vertical slice. Plotting this vector illustrates the profile of total fluorescence intensity with tissue depth, as shown in Fig. 4E. Each single vector thus formed represents total fluorescence intensity distributed along the depth for a single slice. Summing all such vectors (so summing over all vertical slices) yields a total fluorescence intensity distributed along depth for the whole tissue block: $\vec{I}=\sum_{\text {all-slices }} \vec{i}$.

G. Auto-Fluorescence Removal: In order to account for auto-fluorescence in tissue, steps A-E were performed on control samples without particles. The average of all pixel intensities from control 
samples was used as the auto-fluorescence intensity in that tissue type e.g. in liver, kidney or brain. This average pixel intensity of auto-fluorescence was then subtracted from the fluorescence intensity computed in step F.

H. Depth Metric: In order to quantify the distance traveled by the particles in each tissue sample, we computed the centroid of the particle vertical distribution. Let $I_{i}$ represent the total fluorescent intensity at a depth $\mathrm{d}_{\mathrm{i}}$ (representing the $i$ th element of the column vector in $\mathrm{F}$ above). That value was normalized with respect to the maximum intensity at depth $\mathrm{d}_{\mathrm{i}}$ from the top of tissue, and then the centroidal distance that the particles moved was computed as $\boldsymbol{d}_{c}=\sum_{i=1}^{n}\left(\boldsymbol{d}_{\boldsymbol{i}} \cdot \boldsymbol{I}_{\boldsymbol{i}}\right) / \sum_{i=1}^{n} \boldsymbol{I}_{\boldsymbol{i}}$. This distance metric was used for quantifying the movement of the tested particle types in the various tissues.

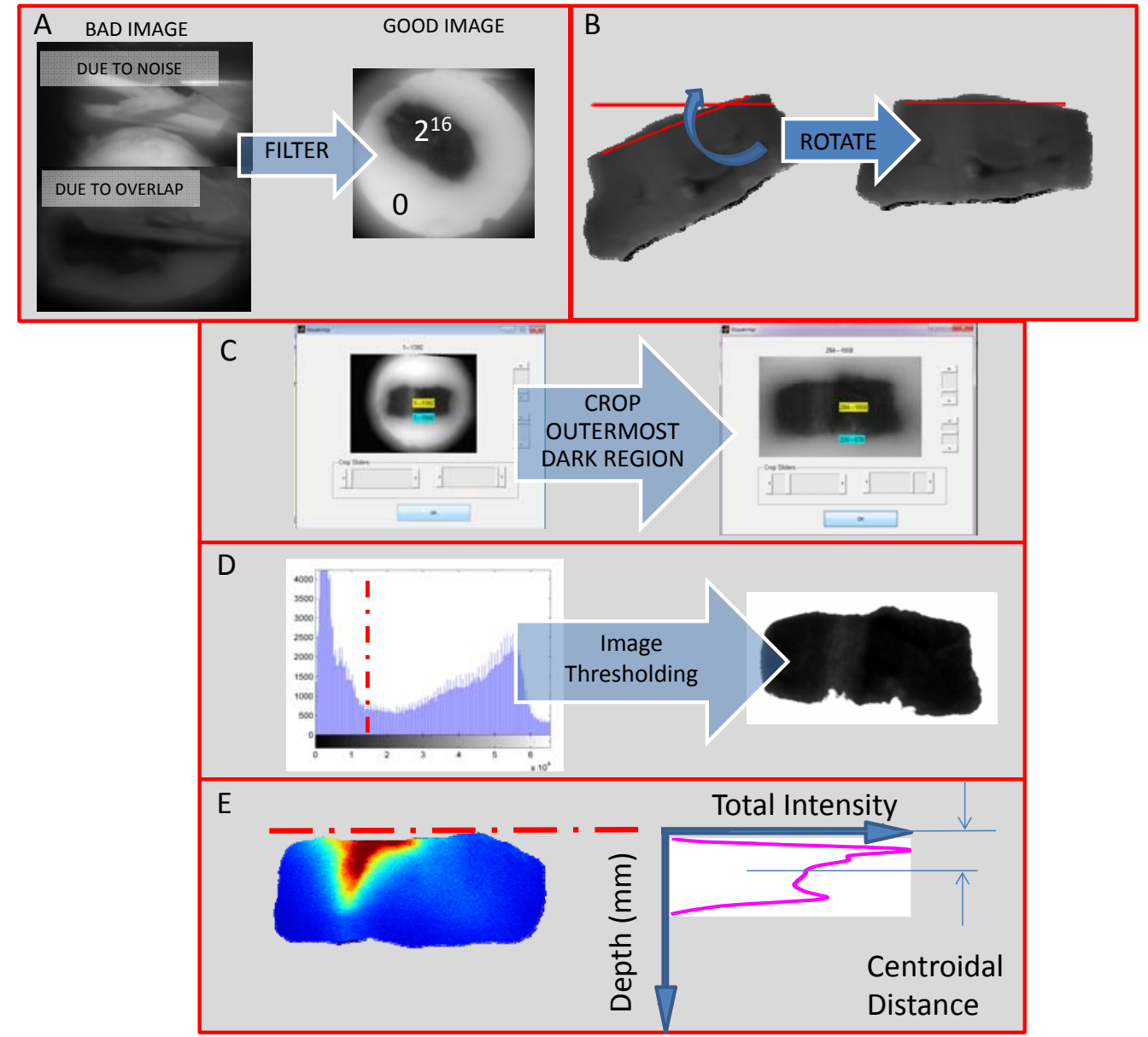

Fig. 4. Image processing steps: (A) A bad image resulting from random capture of an image due to noise or overlapping of a previous slice is removed. This was a rare event, but such images were removed to clean the database. On the right, a good image that is retained is shown. In the good image, the tissue region has pixel values close to zero (dark) and OCT region has pixel values up to 216 (close to white). (B) Tissue images were sometimes not correctly aligned. A typical misalignment was $20^{\circ}$ and usually occurred due to tissue placement error (tissues had to be placed on the cryostat quickly in order to minimize temperature changes). All images were rotated until the top surface appeared horizontal. (C) Dark background was cropped until only white OCT background and dark tissue background was visible, this was done in order to facilitate image processing. (D) The image was thresholded and the bright OCT region was assigned a complete white intensity (255) so that only tissue region was considered for particle measurement. (E) In this coloring, fluorescent particles with high intensities can be seen (red corresponds to a pixel value of 216 , blue corresponds to a pixel value of zero). The sum of all intensities across a single row represents the total number of particles at that depth. The resulting pink curve shows the distribution of particles with depth. The centroid of this pink curve quantifies the depth of penetration of particles into the tissue sample. 


\section{Results and Discussion}

To begin to understand the effect of magnetic field, particle characteristics, and tissue environment on the motion of particles, we studied three types of tissue: liver, kidney and brain, and four types of particles: starch, chitosan, lipid and PEG/P. For each tissue-particle pair, we conducted two types of experiments: 1) we let the particles passively diffuse through the tissue and 2 ) we held a permanent magnet ( $0.4 \mathrm{~T}$ field strength at its surface) immediately below the tissue in order to exert a maximal magnetic force on the particles at the top of the tissue. After conducting the experiments as described in Sec. 2, we measured the fluorescence distribution of particles in tissue samples for each case and computed the centroidal distance $d_{c}$. In Fig. 5 we show representative fluorescent images to illustrate penetration of three different particle types (100 nm chitosan, $100 \mathrm{~nm}$ PEG/P and $300 \mathrm{~nm}$ lipid) in three types of tissue slices (liver, kidney and brain) resulting from passive transport and magnetic drift. As can be seen from Fig. 5, application of the magnet increased particle motion for $100 \mathrm{~nm}$ particles in liver and the brain.

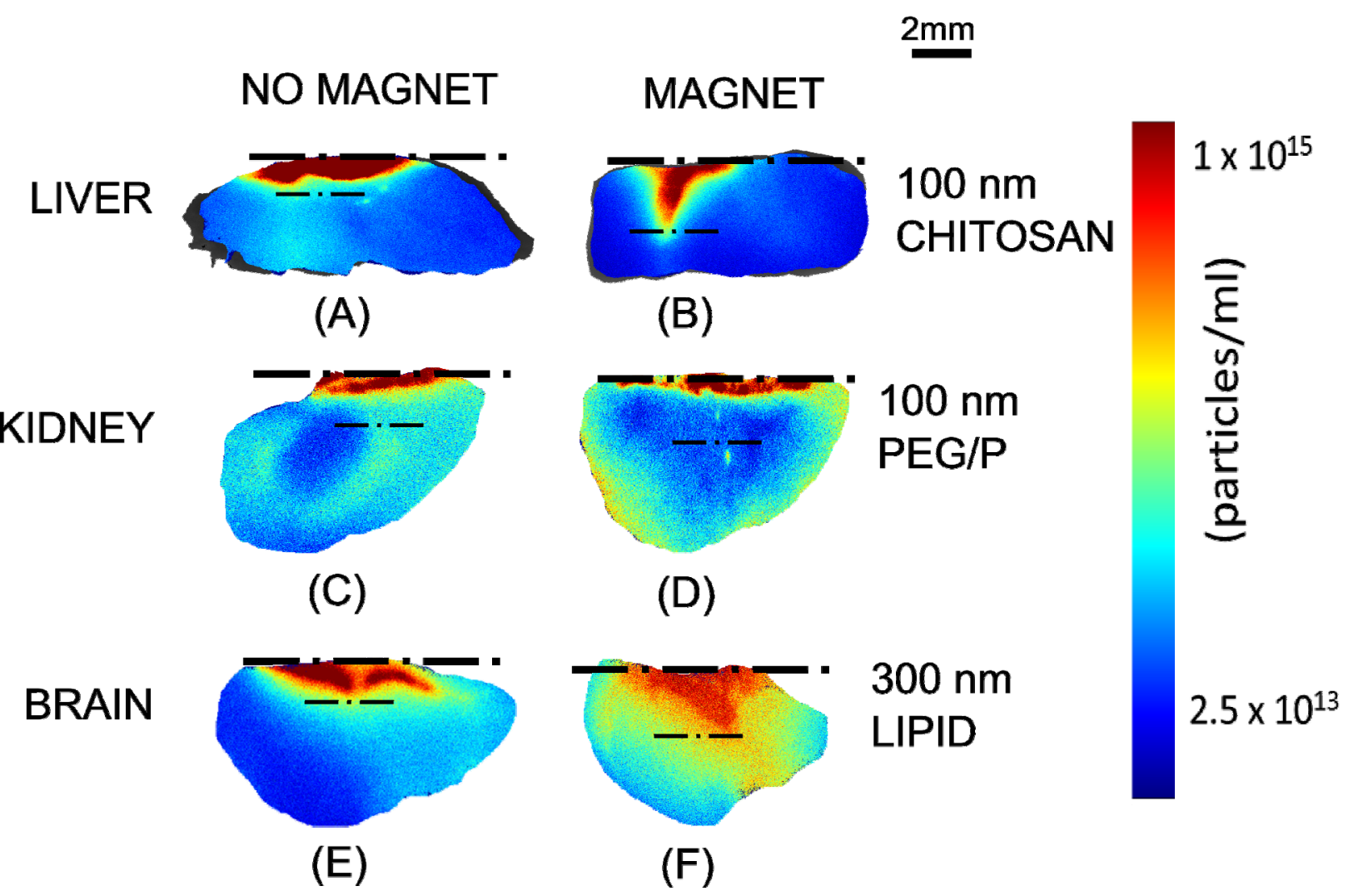

Fig. 5. Magnetic transport of particles versus diffusion alone. The images show fluorescence distribution in three types of particle-tissue pairs for passive and magnetic transport. In each case, dc is centroidal distance that particles penetrate in tissue (de is defined in Sec. 2.5, step $H$ ). Chitosan $100 \mathrm{~nm}$ particles (A) passively diffusing through liver tissue demonstrated limited movement $(\mathrm{dc}=2.6 \mathrm{~mm})$ versus $(\mathrm{B})$ moved substantially when pulled with a magnet $(\mathrm{dc}=5.3 \mathrm{~mm})$. PEG/P $100 \mathrm{~nm}$ particles (C) passively diffused through a section of kidney showing some spreading and diffusion (dc $=2.6$ $\mathrm{mm})$ versus (D) showed some small additional movement when pulled with a magnet $(\mathrm{dc}=3.0 \mathrm{~mm})$. Lipid $300 \mathrm{~nm}$ particles $(E)$ showed limited diffusion in brain tissue $(\mathrm{dc}=2.5 \mathrm{~mm})$ versus $(F)$ substantial magnetic drift $(\mathrm{dc}=5 \mathrm{~mm})$.

The degree of particle penetration into tissue samples under an applied magnetic field was then quantified for each particle type and tissue type pair. Each experiment was repeated three times $(\mathrm{N}=3)$ and the averaged penetration depth and its standard deviation are shown below in Fig. 6. Since our experiments were repeated only three times, we computed coefficient of variation for each experiment. The coefficient 
of variation was 0.25 on average with a maximum of 0.78 and a minimum of 0.038 . We have tabulated the details for each experiment in the Supplementary Materials.

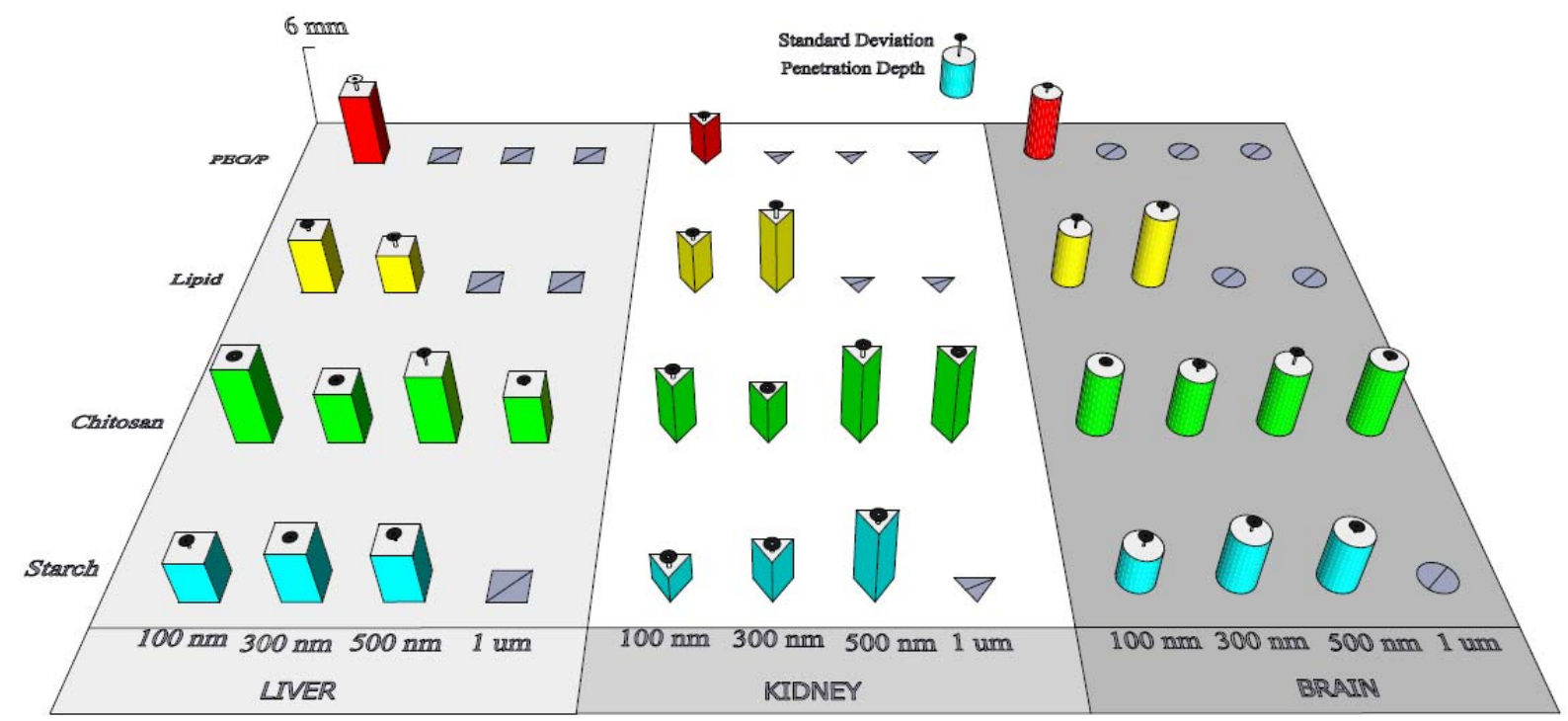

Fig. 6. Tissue penetration after 45 minutes of pulling by a 0.4 Tesla magnet for various particle sizes $(100 \mathrm{~nm}$ to $1 \mu \mathrm{m}$ diameter), with various coating (chitosan, starch, lipid and PEG/P), in different tissue types (rat brain, liver and kidney). Colors denote the type of coating, the shape of the prism denotes the tissue type, and the height of prisms shows the degree of penetration into that tissue. The vertical bar with black disk above it denotes the standard deviation of the measurement. Particles types that were not available from Chemicell are marked by the crossed-out symbols. Source data is tabulated in the Supplementary Data section.

After comparing all available fluorescent Chemicell particle types and their movement through brain, liver, and kidney tissue types, we found that the average particle penetration depth in all three tissue types fell within a range of $1.78 \mathrm{~mm}$ and $5.6 \mathrm{~mm}$ when exposed to a $\sim 0.4$ Tesla magnetic field for 45 minutes. Hence, the average velocity of particle motion in liver, kidney and brain tissue was found to lie between 0.66 and $2 \mu \mathrm{m} / \mathrm{s}$. The particle motion was slowest for $100 \mathrm{~nm}$ starch particles through kidney and was fastest for $1 \mu \mathrm{m}$ chitosan particles in kidney.

We compared the effect of particle coatings on the magnetic drift of particles through tissue. Among all coating types, for available fluorescent particles from Chemicell, we found that chitosan particles (with $+34 \mathrm{mV}$ zeta potential) moved better through the liver, kidney and brain than starch particles (with $-8 \mathrm{mV}$ zeta potential) for particles of all sizes $(100 \mathrm{~nm}$ to $1 \mu \mathrm{m})$. In Fig. 6, the penetration of chitosan particles (green bars) is higher than the penetration of starch particles (blue bars), except for the $300 \mathrm{~nm}$ size where starch particles penetrated slightly deeper than chitosan in liver tissue. Starch particles with a $1 \mu \mathrm{m}$ diameter were not available from Chemicell, as mentioned in Sec.2.1, and are marked by a data-notavailable symbol (crossed-out square, triangle or circle).

Comparison of particle penetration due to magnetic drift versus passive diffusion in all tissue types for all particle sizes tested showed that penetration due to magnetic drift is more effective than passive diffusion for strongly positively charged particles such as chitosan $(+34 \mathrm{mV})$ and strongly negatively charged particles such as PEG/P $(-20 \mathrm{mV})$. The computed mean and standard deviation of centroidal distance penetration by chitosan and PEG/P particles, for magnetic drift versus diffusion, is shown by green and red prisms in Fig. 7. 


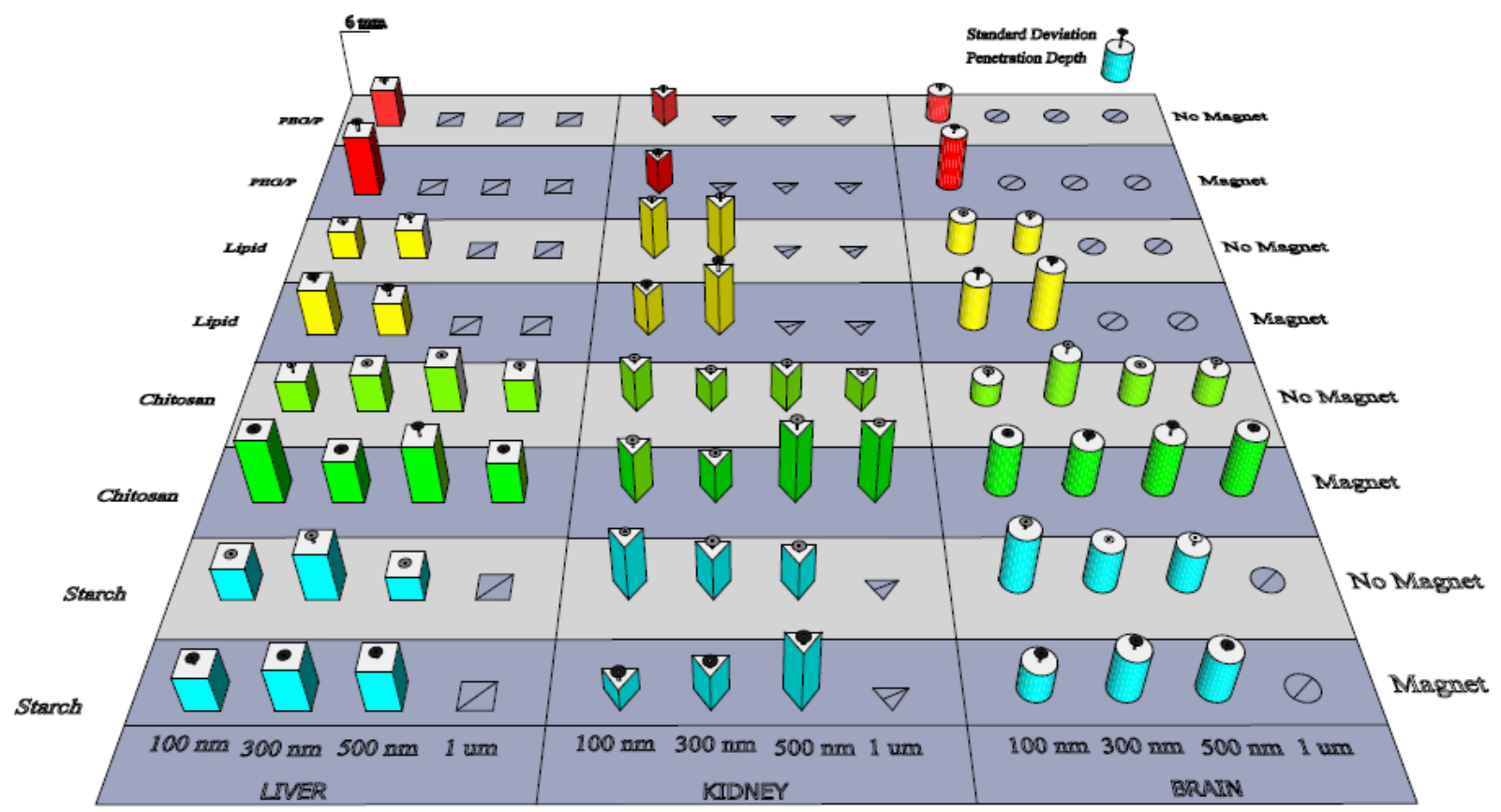

Fig. 7. Tissue penetration after 45 minutes of passive diffusion and pulling by a 0.4 Tesla magnet for all tested particle sizes $(100 \mathrm{~nm}, 300 \mathrm{~nm}, 500 \mathrm{~nm}$ and $1 \mu \mathrm{m})$ and all available coatings (starch represented as blue, chitosan as green, lipid as yellow and PEG/P as red prisms). Penetration depth is represented by the height of each prism. The shape of the prisms represents movement through specific tissue type: square prisms denote movement through liver, triangular prisms denote kidney and cylinders denote brain. The color of the substrate (the base on which the prisms lie) denotes the presence of a pulling magnet (dark gray substrate) versus the absence of a magnet (diffusion only, light substrate). Source data is tabulated in the Supplementary Data section.

Apart from the above clearly discernable trends, we observed some more nuanced effects of particle size on magnetic movement through tissue. Comparison of nanoscreenMag particles (up to $300 \mathrm{~nm}$ in size) showed that the smallest $(100 \mathrm{~nm}$ ) chitosan particles penetrated deeper than $300 \mathrm{~nm}$ size chitosan, in all tissue types. Among screenMag chitosan particles (500 nm size and above), the $500 \mathrm{~nm}$ sized particles penetrated deeper in the liver, penetrated to the same depth in the kidney, and penetrated less deep in the brain, as compared to their $1 \mu \mathrm{m}$ sized counterparts. The largest available $1 \mu \mathrm{m}$ particles with chitosan coating penetrated the deepest through kidney and brain tissues, but this was not the case for motion in liver tissue.

From Fig. 6, we can also observe that penetration of particles due to magnetic drift increased with size for starch particles. This behavior was consistent for all starch particles (sizes $100 \mathrm{~nm}$ to $500 \mathrm{~nm}$ ) in all tissue types. The blue triangular prisms for kidney tissue clearly show increasing penetration depth with starch particle size, although this increase is less pronounced when comparing $300 \mathrm{~nm}$ to $500 \mathrm{~nm}$ starch particles in liver and brain tissues.

We can also compare particle penetration due to magnetic drift for the available $100 \mathrm{~nm}$ and $300 \mathrm{~nm}$ lipid particles in liver, kidney and brain. As shown in Fig. 6, the particle penetration was higher for $300 \mathrm{~nm}$ lipid particles compared to $100 \mathrm{~nm}$ lipid particles in kidney and brain tissue, as shown by the taller yellow triangular prisms and cylinders and their shorter counterparts for $100 \mathrm{~nm}$ particles. However, this behavior was reversed in the case of liver, as shown by the taller $100 \mathrm{~nm}$ yellow square bar compared to the shorter $300 \mathrm{~nm}$ yellow bar in the liver section in Fig. 7. Lipid particles larger than $300 \mathrm{~nm}$ were unavailable (as mentioned in Sec. 2.1) and are marked by a cross-out diagonal within the square, triangle, or circle shapes. 
We made every effort to insure that our results in freshly excised tissue would match in-vivo behavior as closely as possible. The time between organ excision and experiment was less than two hours, and according to accepted tissue handling procedures the tissue was preserved in cool environment $\left(4^{\circ} \mathrm{C}\right)$, and stored in phosphate buffer solution. This procedure and time interval is within the time interval studied in transplant research and research for studying the mechanical properties of tissue ${ }^{38,55-57}$. Further, the tissue organs we selected (liver, kidney, and brain) were significantly larger than the volume of MNPs used, and only the outer surface of the tissue was exposed to the liquefied gelatin used for immobilizing the tissue. This protected the particle penetration path from the external environment to some extent, and we believe it helped ensure that the collected data will be representative of magnetic particle motion in-vivo.

\section{Conclusion}

An experimental method was presented to quantitatively measure the penetration depth of magnetic nanoparticles (MNPs) into tissue samples under the action of an applied magnetic field. In this method, MNPs were placed on top of freshly excised liver, kidney, and brain tissue samples and were then pulled into the samples by a magnet placed underneath the tissues. The tissue samples were sliced by an automated cryostat, fluorescence from the particles was imaged and processed, and the degree of MNP penetration was quantified by a centroid distance metric. Tests were conducted on available fluorescent particles from Chemicell in four sizes $(100 \mathrm{~nm}, 300 \mathrm{~nm}, 500 \mathrm{~nm}$, and $1 \mu \mathrm{m}$ diameter) and with four different coatings (starch, chitosan, lipid, PEG/P). The average particle penetration depth in all three tissue types, after a 45 minute application of a 0.4 Tesla 1" x 1" x 2" magnet, was between $1.78 \mathrm{~mm}$ and $5.6 \mathrm{~mm}$, which corresponds to a transport velocity between 0.66 and $2 \mu \mathrm{m} / \mathrm{s}$. We found that chitosan particles moved most effectively through all three-tissue types (as compared to starch, lipid, and PEG/P coated particles). However, we observed many additional dependencies on particle size, coating, and tissue type, which indicate that the motion of MNPs in tissue is complex and that additional studies will be required to elucidate transport mechanisms in tissue and to engineer MNPs for optimal transport in tissue. We also stress that our data was collected in freshly excised tissue, not in-vivo in live animals, and differences between excised and living tissue may affect MNP motion in ways that are not captured by this study.

\section{Acknowledgments}

Funding from the National Institutes of Health (NIH), the National Science Foundation (NSF), and the CDMRP, as well as the Technology Development Corporation (TEDCO) and the Maryland Industrial Partnerships (MIPS) agencies in the State of Maryland and Action on Hearing Loss in the United Kingdom, is gratefully acknowledged.

\section{References}

1. Alexiou, C. et al. Magnetic Drug Targeting - A new approach in locoregional tumortherapy with chemotherapeutic agents. Experimental animal studies. Hno 53, 618-622 (2005).

2. Johannsen, M. et al. Clinical hyperthermia of prostate cancer using magnetic nanoparticles: Presentation of a new interstitial technique. Int. J. Hyperthermia 21, 637-647 (2005).

3. Weissleder, R. et al. In vivo magnetic resonance imaging of transgene expression. Nat. Med. 6, 351-354 (2000).

4. Bonnemain, B. Superparamagnetic agents in magnetic resonance imaging: physicochemical characteristics and clinical applications. A review. J. Drug Target. 6, 167-174 (1998). 
5. Nacev, A., Beni, C., Bruno, O. \& Shapiro, B. The Behaviors of Ferro-Magnetic Nano-Particles In and Around Blood Vessels under Applied Magnetic Fields. J. Magn. Magn. Mater. 323, 651-668 (2011).

6. Nacev, A., Beni, C., Bruno, O. \& Shapiro, B. Magnetic Nanoparticle Transport within Flowing Blood and Into Surrounding Tissue. Nanomed. 5, 1459-66 (2010).

7. Nacev, A. et al. Towards Control of Magnetic Fluids in Patients: Directing Therapeutic Nanoparticles to Disease Locations. IEEE Control Syst. 32, 32-74 (2012).

8. Pankhurst, Q. A., Thanh, N. T. K., Jones, S. K. \& Dobson, J. Progress in applications of magnetic nanoparticles in biomedicine. J. Phys. Appl. Phys. 42, 224001 (2009).

9. Dobson, J. Magnetic micro- and nano-particle-based targeting for drug and gene delivery. Nanomed. 1, 31-37 (2006).

10. Lubbe, A. S., Alexiou, C. \& Bergemann, C. Clinical applications of magnetic drug targeting. J. Surg. Res. 95, 200-206 (2001).

11. Shapiro, B. et al. Shaping Magnetic Fields to Direct Therapy to Ears and Eyes. Annu. Rev. Biomed. Eng. (2014). doi:10.1146/annurev-bioeng-071813-105206

12. Alexiou, C. et al. Targeting cancer cells: magnetic nanoparticles as drug carriers. Eur. Biophys. J. Biophys. Lett. 35, 446-450 (2006).

13. Sun, C., Lee, J. S. H. \& Zhang, M. Magnetic nanoparticles in MR imaging and drug delivery. Adv. Drug Deliv. Rev. 60, 1252-1265 (2008).

14. Yigit, M. V., Moore, A. \& Medarova, Z. Magnetic nanoparticles for cancer diagnosis and therapy. Pharm. Res. 29, 1180-1188 (2012).

15. Brigger, I., Dubernet, C. \& Couvreur, P. Nanoparticles in cancer therapy and diagnosis. Adv Drug Deliv Rev 54, 631-651 (2002).

16. McCarthy, J. R. \& Weissleder, R. Multifunctional magnetic nanoparticles for targeted imaging and therapy. Adv. Drug Deliv. Rev. 60, 1241-1251 (2008).

17. Mornet, S., Vasseur, S., Grasset, F. \& Duguet, E. Magnetic nanoparticle design for medical diagnosis and therapy. J. Mater. Chem. 14, 2161-2175 (2004).

18. Lai, S. K. et al. Rapid transport of large polymeric nanoparticles in fresh undiluted human mucus. Proc. Natl. Acad. Sci. 104, 1482-1487 (2007).

19. Kalambur, V. S., Han, B., Hammer, B. E., Shield, T. W. \& Bischof, J. C. In vitro characterization of movement, heating and visualization of magnetic nanoparticles for biomedical applications. Nanotechnology 16, 1221 (2005). 
20. Allard, E., Passirani, C. \& Benoit, J.-P. Convection-enhanced delivery of nanocarriers for the treatment of brain tumors. Biomaterials 30, 2302-2318 (2009).

21. MacKay, J. A., Deen, D. F. \& Szoka Jr., F. C. Distribution in brain of liposomes after convection enhanced delivery; modulation by particle charge, particle diameter, and presence of steric coating. Brain Res. 1035, 139-153 (2005).

22. Thorne, R. G. \& Nicholson, C. In vivo diffusion analysis with quantum dots and dextrans predicts the width of brain extracellular space. Proc. Natl. Acad. Sci. 103, 5567-5572 (2006).

23. Nance, E. A. et al. A Dense Poly(Ethylene Glycol) Coating Improves Penetration of Large Polymeric Nanoparticles Within Brain Tissue. Sci. Transl. Med. 4, 149ra119-149ra119 (2012).

24. Lee, J.-C. \& Lee, S. Dielectrophoresis-magnetophoresis force driven magnetic nanoparticle movement in transformer oil based magnetic fluids. J. Nanosci. Nanotechnol. 13, 6179-6182 (2013).

25. Valberg, P. A. \& Feldman, H. A. Magnetic particle motions within living cells. Measurement of cytoplasmic viscosity and motile activity. Biophys. J. 52, 551-561 (1987).

26. Zhang, E. et al. Dynamic Magnetic Fields Remote-Control Apoptosis via Nanoparticle Rotation. ACS Nano 8, 3192-3201 (2014).

27. Baroli, B. et al. Penetration of Metallic Nanoparticles in Human Full-Thickness Skin. J Invest Dermatol 127, 1701-1712 (2007).

28. Pankhurst, Q. A., Connolly, J., Jones, S. K. \& Dobson, J. Applications of magnetic nanoparticles in biomedicine. J. Phys. Appl. Phys. 36, R167 (2003).

29. Domínguez-García, P., Melle, S., Pastor, J. M. \& Rubio, M. A. Scaling in the aggregation dynamics of a magnetorheological fluid. Phys. Rev. E 76, 051403 (2007).

30. Sano, K. \& Doi, M. Theory of agglomeration of ferromagnetic particles in magnetic fluids. J PHYS SOC Jpn. 52, 2810-2815 (1983).

31. Taketomi, S., Takahashi, H., Inaba, N. \& Miyajima, H. Experimental and theoretical investigations on agglomeration of magnetic colloidal particles in magnetic fluids. J Phys Soc Jpn 60, 1689-1707 (1991).

32. Saltzman, W. M. Drug Delivery: Engineering Principles for Drug Therapy. (Oxford University Press, 2001).

33. Bausch, A. R., Ziemann, F., Boulbitch, A. A., Jacobson, K. \& Sackmann, E. Local Measurements of Viscoelastic Parameters of Adherent Cell Surfaces by Magnetic Bead Microrheometry. Biophys. J. 75, 2038-2049 (1998).

34. Valberg, P. A. \& Albertini, D. F. Cytoplasmic motions, rheology, and structure probed by a novel magnetic particle method. J. Cell Biol. 101, 130-140 (1985). 
35. Renkin, E. M. Filtration, diffusion, and molecular sieving through porous cellulose membranes. J. Gen. Physiol. 38, 225-243 (1954).

36. Ogston, A. G., Preston, B. N. \& Wells, J. D. On the transport of compact particles through solutions of chain-polymers. Proc. R. Soc. Lond. Math. Phys. Sci. 333, 297 (1973).

37. Nacev, A. et al. A dynamic magnetic shift method to increase nanoparticle concentration in cancer metastases: a feasibility study using simulations on autopsy specimens. Int. J. Nanomedicine 6, 2907-2923 (2011).

38. Fung, Y.-C. Biomechanics. (Springer New York, 1993). at <http://link.springer.com/10.1007/9781-4757-2257-4>

39. Ikada, Y. Tissue Engineering: Fundamentals and Applications. (Academic Press, 2011).

40. Teicher, B. A. In Vivo/Ex Vivo and In Situ Assays Used in Cancer Research: A Brief Review. Toxicol. Pathol. 37, 114-122 (2009).

41. Zhaolong Shen et al. Automated Fluorescence and Reflectance Coregistered 3-D Tissue Imaging System. Magn. IEEE Trans. On 49, 279-284 (2013).

42. Fleisch, D. A. A Student's Guide to Maxwell's Equations. (Cambridge University Press, 2008).

43. Forbes, Z. G., Yellen, B. B., Barbee, K. A. \& Friedman, G. An approach to targeted drug delivery based on uniform magnetic fields. IEEE Trans. Magn. 39, 3372-3377 (2003).

44. Forbes, Z. G. et al. Validation of high gradient magnetic field based drug delivery to magnetizable implants under flow. IEEE Trans. Biomed. Eng. 55, 643-649 (2008).

45. Jackson, J. D. Classical Electrodynamics Third Edition. (Wiley, 1998).

46. Feynman, R. P. Feynman lectures on physics. (Addison Wesley Longman, 1970).

47. Mikkelsen, C. I. Magnetic separation and hydrodynamic interactions in microfluidic systems. (Technical University of Denmark, 2005).

48. Foner, S. Versatile and Sensitive Vibrating \#x2010;Sample Magnetometer. Rev. Sci. Instrum. 30, 548-557 (1959).

49. Murdock, R. C., Braydich-Stolle, L., Schrand, A. M., Schlager, J. J. \& Hussain, S. M. Characterization of Nanomaterial Dispersion in Solution Prior to In Vitro Exposure Using Dynamic Light Scattering Technique. Toxicol. Sci. 101, 239-253 (2008).

50. Seliger, C. et al. In vitro investigation of the behaviour of magnetic particles by a circulating artery model. J. Magn. Magn. Mater. 311, 358-362 (2007). 
51. Bootz, A., Vogel, V., Schubert, D. \& Kreuter, J. Comparison of scanning electron microscopy, dynamic light scattering and analytical ultracentrifugation for the sizing of poly(butyl cyanoacrylate) nanoparticles. Eur. J. Pharm. Biopharm. 57, 369-375 (2004).

52. Sennett, P. \& Olivier, J. P. COLLOIDAL DISPERSIONS, ELECTROKINETIC EFFECTS, AND THE CONCEPT OF ZETA POTENTIAL. Ind. Eng. Chem. 57, 32-50 (1965).

53. Zhang, Y. et al. Zeta potential: a surface electrical characteristic to probe the interaction of nanoparticles with normal and cancer human breast epithelial cells. Biomed. Microdevices 10, 321-328 (2007).

54. Verwey, E. J. W., Overbeek, J. T. G. \& Overbeek, J. T. G. Theory of the Stability of Lyophobic Colloids. (Courier Dover Publications, 1999).

55. Samani, A. \& Plewes, D. A method to measure the hyperelastic parameters of ex vivo breast tissue samples. Phys. Med. Biol. 49, 4395 (2004).

56. Belzer Fo, Glass Nr, Sollinger Hw, Hoffmann Rm \& Southard Jh. A new perfusate for kidney preservation. Transplantation 33, 322-323 (1982).

57. Collins, G. M., Bravo-Shugarman, M. \& Terasaki, P. I. KIDNEY PRESERVATION FOR

TRANSPORTATION. The Lancet 294, 1219-1222 (1969). 


\section{Particle Distribution Data}

Particle distribution plots were acquired using the software for the Photocor Dynamic Light Scattering System. The distribution shows hydrodynamic radius of particles, and is fairly Gaussian. In Fig. 8, the particle distribution, for each particle type (size and coating) is shown, and Fig. 9 shows polymeric particles of size $100 \mathrm{~nm}$ and $300 \mathrm{~nm}$, used as NIST standards.
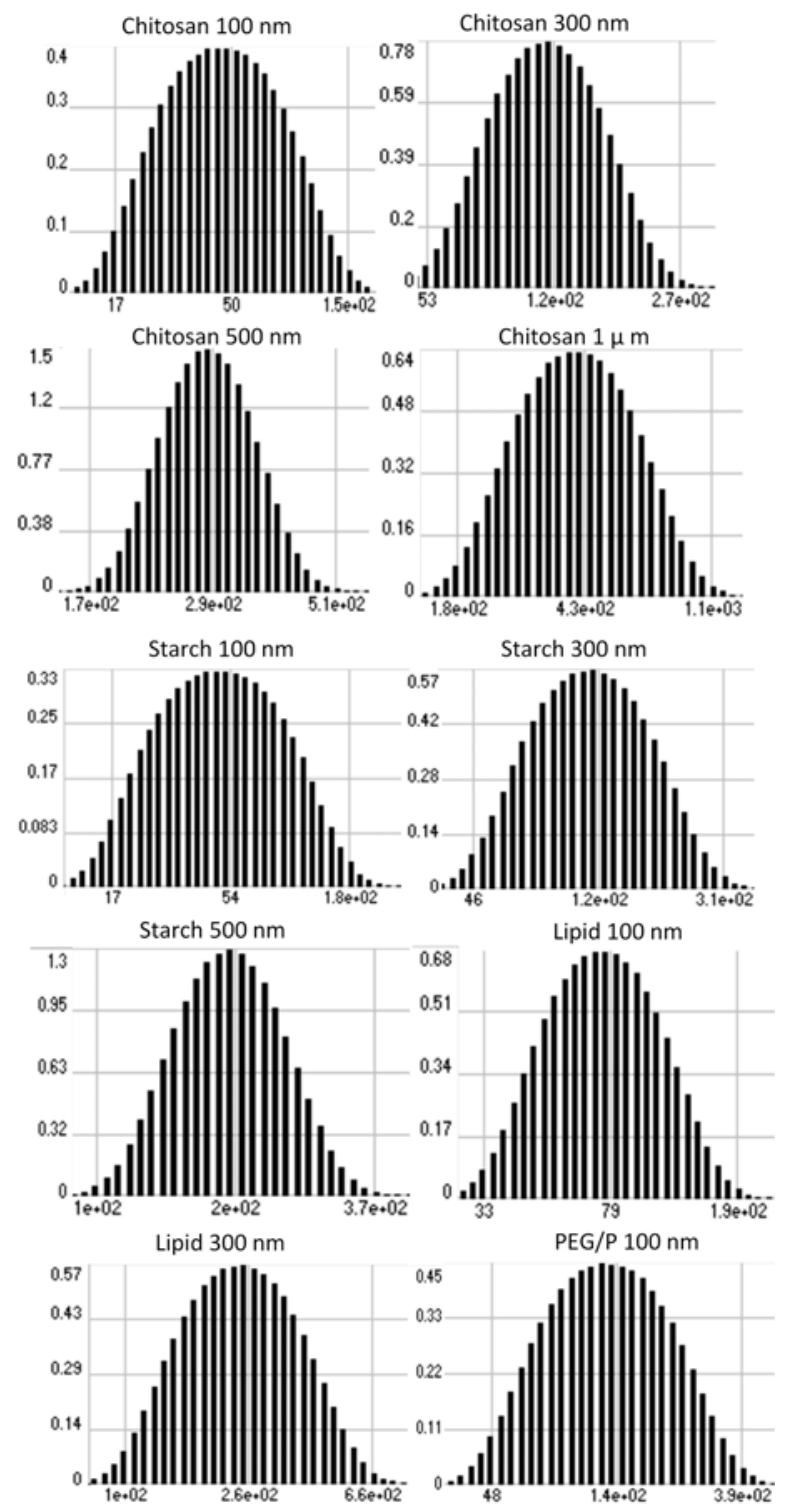

Fig. 8 Particle distribution plots showing intensity vs particle hydrodynamic radius, based on DLS measurements using Photocor Instruments are shown above. The hydrodynamic radius of MNPs matches closely with the specified radius from Chemicell. Note that $300 \mathrm{~nm}$ Lipid and $100 \mathrm{~nm}$ PEG/P particles had larger size according to DLS measurements, possibly due to agglomeration. 

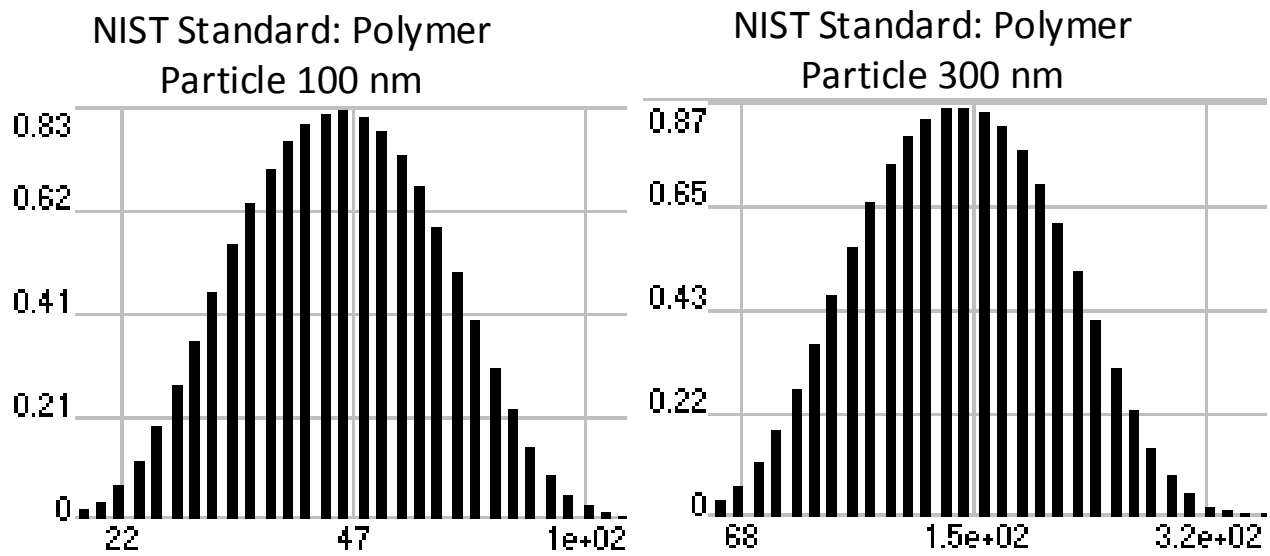

Fig. 9 Particle distribution plots showing intensity vs particle hydrodynamic radius for NIST polymer particles of size 100 $\mathrm{nm}$ and $300 \mathrm{~nm}$ diameter, based on DLS measurements using Photocor Instruments are shown above.

\section{Tables of Particle Penetration Depths}

In this section, we provide tabulated experimental results for the figures provided in the main text. Table 5 lists particle penetration for all particle types (size and coating) in all tissue types after 45 minutes of magnetic pulling. Table 6 lists particle penetration for all particle types (size and coating) in all tissue types after 45 minutes of passive diffusion. Table 7 and 8 show the coefficient of variations for Table 5 and 6 respectively.

\begin{tabular}{|c|c|c|c|c|c|c|c|c|c|c|c|c|}
\hline \multicolumn{13}{|c|}{ Active Transport of Particles In Tissue (in mm after 45 minutes) } \\
\hline \multicolumn{5}{|c|}{ BRAIN } & \multicolumn{4}{|c|}{ LIVER } & \multicolumn{4}{|c|}{ KIDNEY } \\
\hline Zeta $(\mathrm{mV})$ & 34 & -8 & -19 & -20 & 34 & -8 & -19 & -20 & 34 & -8 & -19 & -20 \\
\hline Size Coating & Chitosan & Starch & Lipid & PEG/P & Chitosan & Starch & Lipid & PEG/P & Chitosan & Starch & Lipid & PEG/P \\
\hline $100 \mathrm{~nm}$ & $4.3 \pm 0.5$ & $2.4 \pm 1.4$ & $3.8 \pm 1.5$ & $4.7 \pm 1$ & $5.3 \pm 0.2$ & $2.86 \pm 1$ & $4 \pm 0.9$ & $5.2 \pm 1.6$ & $4.1 \pm 1.2$ & $1.78 \pm 1.4$ & $3.5 \pm 1$ & $3.0 \pm 0.8$ \\
\hline $300 \mathrm{~nm}$ & $3.9 \pm 1.0$ & $3.5 \pm 1.3$ & $5.0 \pm 0.9$ & & $3.6 \pm 0.2$ & $3.9 \pm 1.2$ & $2.8 \pm 1.4$ & & $3.1 \pm 0.5$ & $2.9 \pm 1$ & $5.1+1.8$ & \\
\hline $500 \mathrm{~nm}$ & $4.4 \pm 1.7$ & $3.4 \pm 0.8$ & & & $4.8 \pm 2.1$ & $3.4 \pm 0.9$ & & & $5.6 \pm 1.6$ & $4.9 \pm 1.1$ & & \\
\hline \multirow[t]{5}{*}{1 micron } & $4.7 \pm 0.6$ & & & & $3.4 \pm 0.2$ & & & & $5.6 \pm 0.6$ & & & \\
\hline & & & & & & & & & & & & \\
\hline & \multicolumn{3}{|c|}{ Relatively low } & $<2.5$ & & & High & & $4.5-5.25$ & & \multicolumn{2}{|c|}{ mean \pm std dev. } \\
\hline & \multicolumn{3}{|c|}{ Moderate } & $2.5--3.5$ & & & \multicolumn{2}{|l|}{ Very High } & $>5.25$ & & & \\
\hline & \multicolumn{3}{|c|}{ Relatively high } & 3.5--4.5 & & & \multicolumn{4}{|c|}{ Not available from Chemicell } & & \\
\hline
\end{tabular}

Table 5: Tissue penetration after 45 minutes of pulling by a 0.4 Tesla magnet for various particle sizes $(100 \mathrm{~nm}-1 \mu \mathrm{m}$ diameter), coating (chitosan, starch, lipid and PEG/P), and tissue types (rat brain, liver and kidney). Colors denote degree of penetration into the tissue, from red (low penetration) to green (high), as noted in the legend at the bottom. 


\begin{tabular}{|c|c|c|c|c|c|c|c|c|c|c|c|c|}
\hline \multicolumn{13}{|c|}{ Passive Transport of Particles In Tissue (in $\mathrm{mm}$ after 45 minutes) } \\
\hline \multicolumn{5}{|c|}{ BRAIN } & \multicolumn{4}{|c|}{ LIVER } & \multicolumn{4}{|c|}{ KIDNEY } \\
\hline Zeta $(\mathrm{mV})$ & 34 & & -19 & -8 & 34 & -8 & -19 & -20 & 34 & & -19 & -8 \\
\hline Size Coating & Chitosan & Starch & Lipid & PEG/P & Chitosan & Starch & Lipid & PEG/P & Chitosan & Starch & Lipid & PEG/P \\
\hline $100 \mathrm{~nm}$ & $1.8 \pm 0.9$ & $3.5 \pm 1.1$ & $2.8 \pm 1.3$ & $2.5 \pm 0.8$ & $2.6 \pm 1.3$ & $2.7 \pm 0.4$ & $2.4 \pm 0.7$ & $3.4 \pm 0.8$ & $3.4 \pm 0.9$ & $4.5 \pm 0.6$ & $4.4 \pm 0.6$ & $2.6 \pm 0.8$ \\
\hline $300 \mathrm{~nm}$ & $3.8 \pm 1.5$ & $3.2 \pm 0.35$ & $2.5 \pm 0.5$ & & $3.2 \pm 1$ & $3.9 \pm 1.2$ & $2.6 \pm 1.2$ & & $2.5 \pm 0.7$ & $3.4 \pm 1.2$ & $4.5 \pm 0.9$ & \\
\hline $500 \mathrm{~nm}$ & $2.7 \pm 0.2$ & $3.8 \pm 1.2$ & & & $3.9 \pm 0.4$ & $2.0 \pm 0.26$ & & & $3.0 \pm 0.8$ & $3.1 \pm 0.96$ & & \\
\hline \multirow[t]{5}{*}{1 micron } & $2.5 \pm 1.2$ & & & & $2.8 \pm 0.8$ & & & & $2.4 \pm 0.4$ & & & \\
\hline & & & & & & & & & & & & \\
\hline & \multicolumn{3}{|c|}{ Relatively low } & $<2.5$ & & & High & & $4.5-5.25$ & & \multicolumn{2}{|c|}{ mean \pm std dev. } \\
\hline & \multicolumn{3}{|c|}{ Moderate } & $2.5--3.5$ & & & \multicolumn{2}{|l|}{ Very High } & $>5.25$ & & & \\
\hline & \multicolumn{3}{|c|}{ Relatively high } & $3.5--4.5$ & & & \multicolumn{3}{|c|}{ Unavailable from Chemicell } & & & \\
\hline
\end{tabular}

Table 6: Tissue penetration after 45 minutes of passive transport for various particle sizes $(100 \mathrm{~nm}-1 \mu \mathrm{m}$ diameter), coating (chitosan, starch, lipid and PEG/P), and tissue types (rat brain, liver and kidney) is shown above. Colors denote degree of penetration into the tissue, from red (low penetration) to green (high), as noted in the legend at the bottom.

\begin{tabular}{|c|c|c|c|c|c|c|c|c|c|c|c|c|}
\hline \multicolumn{13}{|c|}{ Coefficient of Variation for Active Transport of Particles In Tissue (in mm after 45 minutes) } \\
\hline \multicolumn{5}{|c|}{ BRAIN } & \multicolumn{4}{|c|}{ LIVER } & \multicolumn{4}{|c|}{ KIDNEY } \\
\hline Zeta $(\mathrm{mV})$ & 34 & -8 & -19 & -20 & 34 & -8 & -19 & -20 & 34 & -8 & -19 & -20 \\
\hline Size Coating & Chitosan & Starch & Lipid & PEG/P & Chitosan & Starch & Lipid & PEG/P & Chitosan & Starch & Lipid & PEG/P \\
\hline $100 \mathrm{~nm}$ & 0.116 & 0.583 & 0.395 & 0.213 & 0.038 & 0.350 & 0.225 & 0.308 & 0.293 & 0.787 & 0.286 & 0.267 \\
\hline $300 \mathrm{~nm}$ & 0.256 & 0.371 & 0.180 & & 0.056 & 0.308 & 0.500 & & 0.161 & 0.345 & 0.353 & \\
\hline $500 \mathrm{~nm}$ & 0.386 & 0.235 & & & 0.438 & 0.265 & & & 0.286 & 0.224 & & \\
\hline \multirow[t]{5}{*}{1 micron } & 0.128 & & & & 0.059 & & & & 0.107 & & & \\
\hline & & & & & & & & & & & & \\
\hline & \multicolumn{3}{|c|}{$<0.2$} & & & & High & & $<0.8$ & \multicolumn{3}{|c|}{ Coefficient of Variation } \\
\hline & \multicolumn{3}{|c|}{$<0.4$} & & & & \multicolumn{2}{|l|}{ Very High } & & \multicolumn{2}{|c|}{ Std.Dev/Mean } & \\
\hline & \multicolumn{3}{|c|}{$<0.6$} & & & & \multicolumn{3}{|c|}{ Unavailable from Chemicell } & & & \\
\hline
\end{tabular}

Table 7: Coefficient of variation for $\mathrm{N}=3$ experiments showing dispersion in tissue penetration after 45 minutes of pulling by a 0.4 Tesla magnet for various particle sizes $(100 \mathrm{~nm}-1 \mu \mathrm{m}$ diameter), coating (chitosan, starch, lipid and PEG/P), and tissue types (rat brain, liver and kidney). Colors denote amount of coefficient of variation of penetration into the tissue, from red (low) to green (high), as noted in the legend at the bottom. On an average the coefficient of variation was 0.25 with a maximum of 0.78 and minimum of 0.038 .

\begin{tabular}{|c|c|c|c|c|c|c|c|c|c|c|c|c|}
\hline \multicolumn{13}{|c|}{ Coefficient of Variation for Passive Transport of Particles In Tissue (in mm after 45 minutes) } \\
\hline \multicolumn{5}{|c|}{ BRAIN } & \multicolumn{4}{|c|}{ LIVER } & \multicolumn{4}{|c|}{ KIDNEY } \\
\hline Zeta $(\mathrm{mV})$ & 34 & & -19 & -8 & 34 & -8 & -19 & -20 & 34 & & -19 & -8 \\
\hline Size Coating & Chitosan & Starch & Lipid & PEG/P & Chitosan & Starch & Lipid & PEG/P & Chitosan & Starch & Lipid & PEG/P \\
\hline $100 \mathrm{~nm}$ & 0.500 & 0.314 & 0.464 & 0.320 & 0.500 & 0.148 & 0.292 & 0.235 & 0.265 & 0.133 & 0.136 & 0.308 \\
\hline $300 \mathrm{~nm}$ & 0.395 & 0.109 & 0.200 & & 0.313 & 0.308 & 0.462 & & 0.280 & 0.353 & 0.200 & \\
\hline $500 \mathrm{~nm}$ & 0.074 & 0.316 & & & 0.103 & 0.130 & & & 0.267 & 0.310 & & \\
\hline \multirow[t]{5}{*}{1 micron } & 0.480 & & & & 0.286 & & & & 0.167 & & & \\
\hline & & & & & & & & & & & & \\
\hline & \multicolumn{3}{|c|}{$<0.2$} & & & & High & & $<0.8$ & \multicolumn{3}{|c|}{ Coefficient of Variation } \\
\hline & \multicolumn{3}{|c|}{$<0.4$} & & & & \multicolumn{2}{|l|}{ Very High } & & \multicolumn{2}{|c|}{ Std.Dev/Mean } & \\
\hline & \multicolumn{3}{|c|}{$<0.6$} & & & & \multicolumn{3}{|c|}{ Unavailable from Chemicell } & & & \\
\hline
\end{tabular}

Table 8: Coefficient of variation for $\mathrm{N}=3$ experiments showing dispersion in tissue penetration after 45 minutes of passive transport for various particle sizes $(100 \mathrm{~nm}-1 \mu \mathrm{m}$ diameter), coating (chitosan, starch, lipid and PEG/P), and tissue types (rat brain, liver and kidney). Colors denote amount of coefficient of variation of penetration into the tissue, from red (low) 
to green (high), as noted in the legend at the bottom. On an average the coefficient of variation was 0.27 with a maximum of 0.5 and a minimum of 0.07 . 